\title{
Estrategias de posicionamiento del Autor en Artículos de Investigación de Ciencias Sociales, Humanidades e Ingeniería: Novatos versus Expertos
}

\author{
Paulina Meza \\ Departamento de Artes y Letras, Universidad de La Serena, Benavente 1140, La Serena, Chile. \\ (e-mail: pmeza@userena.cl)
}

Recibido Ago. 7, 2017; Aceptado Sep. 28, 2017; Versión final Nov. 25, 2017, Publicado Abr. 2018

\begin{abstract}
Resumen
El objetivo de esta investigación es analizar la variación del uso de estrategias de posicionamiento del autor en artículos de investigación de Ciencias Sociales, Humanidades e Ingeniería, producidos por autores únicos novatos y expertos. Se realizó un estudio mixto, en el que, con un método inductivo-deductivo, se analizó un corpus representativo, constituido por 165 artículos, etiquetado con 2956 estrategias. El modelo de posicionamiento está constituido por categorías de análisis propias, validadas exitosamente en distintas etapas. Los resultados muestran que en todas las áreas y grados de experticia se posiciona prioritariamente el aporte al conocimiento, luego las características del investigador y, en último lugar, las características del texto. Los autores de Ingeniería son quienes más priorizan este aporte al conocimiento. Como conclusión central destacamos que son los autores y áreas que necesitan ganar reconocimiento quienes mayor cantidad de estrategias de posicionamiento utilizan. Estos son, los autores novatos y el área de Ciencias Sociales.
\end{abstract}

Palabras clave: análisis del discurso científico; Artículo de Investigación; posicionamiento estratégico del autor; áreas de la ciencia; grado de experticia

\section{Author Positioning Strategies in Research Articles of Social Science, Humanities and Engineering: Experts versus Novices}

\begin{abstract}
This study aims at analyzing how positioning strategies of expert and novice single authors vary in research articles from Social Science, Humanities and Engineering. An inductive-deductive method was used over a corpus consisting of 165 articles coded with 2956 strategies. Our positioning model consists of original analytical categories which were validated throughout different stages. The results showed that the contribution to knowledge strategy predominates in all fields and levels of expertise, followed by the characteristics of the researcher and the features of the text. The authors belonging to Engineering are the ones who gave more priority to the contribution to knowledge. As a conclusion, we highlight that authors and fields requiring more acknowledgement use more positioning strategies. These are, the novice authors and the Social Sciences area.
\end{abstract}

Keywords: scientific discourse analysis; research article; strategic author positioning; scientific fields;

levels of expertise 


\section{INTRODUCCIÓN}

El Artículo de Investigación Científica (en adelante, AIC) se ha transformado en el género fundamental para la difusión del conocimiento científico, por lo que, como se ha señalado previamente (Meza, 2016), se ha convertido en objeto de estudio de una cantidad significativa de investigadores (Li y Ge, 2009; Loi, 2010; Sabaj, Matsuda y Fuenes, 2010; Basturkmen, 2012; entre muchos otros). Si bien los numerosos analistas que han abordado el estudio del AIC lo han hecho desde distintos puntos de vista, en distintas lenguas y a partir de metodologías diversas, en términos generales, es posible clasificar dichas investigaciones en dos grandes grupos, mencionados en Meza (2017d). Por una parte, se encuentran los trabajos que conciben el AIC como un género neutro, transparente, que debe estar libre de todo tipo de expresividad y marcas personales (Sánchez, 2011); y que, además, se caracterizaría por un ocultamiento de la presencia del autor (Müller, 2007) y por la utilización de un lenguaje neutro, preciso y económico (Gutiérrez, 2005). Estas afirmaciones responden a una visión parcial, ingenua y poco realista del AIC, pues no revelan que el acto de escribir y publicar un AIC es una acción estratégico-persuasiva. Así, no basta con presentar y describir resultados, sino que también es fundamental persuadir a la audiencia de su relevancia y pertinencia, de que merecen ser leídos y considerados por la comunidad (Meza, 2016).

El segundo grupo mencionado corresponde a aquellas investigaciones que se apartan de esta concepción objetivizante del AIC y que han indagado, por ejemplo, en la identidad autorial (Abdi y Farrokhi, 2015; Yotimart y Aziz, 2017, entre otros); enfatizadores, mitigadores y marcadores de actitud (Hu y Cao, 2011); metadiscurso (Kim y Lim, 2013; Estaji y Vafaeimehr, 2015; entre otros), inclusión de la voz del autor (Jiang, 2017), etc. Si bien estos trabajos han contribuido de manera sustantiva a evidenciar la presencia del autor en el discurso científico y a cuestionar la concepción del AIC como un género objetivo e impersonal, son investigaciones de tipo más bien léxicogramatical y de análisis de partículas o del discurso. Ello implica que se han dedicado a identificar rasgos particulares de la presencia del autor como, por ejemplo, utilización de la primera persona plural para mostrar identidad, uso de preguntas retóricas, verbos de evidencialidad, marcadores metadiscursivos, etc. (Meza, 2017d).

Ante este panorama, y a fin de poner en primer plano la dimensión estratégica y persuasiva del AIC que otros estudios desconocen, se ha propuesto la noción de posicionamiento estratégico del autor (Meza, 2016, 2017a). Ella ha sido elaborada a partir del complemento entre una indagación teórico-bibliográfica (Meza, 2016) y el estudio empírico de un corpus representativo de una población mayor (Meza, 2017a). Sobre la base de ellos, se ha definido el posicionamiento estratégico del autor como un acto discursivo complejo de carácter persuasivo y argumentativo, que permite dar cuenta de la forma en que un investigador se presenta y se valora a sí mismo; y cómo expone y valora su investigación, en sus escritos, frente a una audiencia (Meza, 2016). A partir de una investigación empírica, se ha descubierto, además, que el posicionamiento estratégico del autor supone dos funciones discursivas generales, a saber, valorar positivamente lo propio y negativamente lo ajeno. Estas macrofunciones se concretizan en estrategias específicas, las que, a su vez, se agrupan en tres dimensiones: epistémica, social y textual (Meza, 2017a).

La dimensión epistémica incluye aquellas estrategias que el autor de un AIC utiliza para posicionar y valorar positivamente su investigación como una contribución al campo de conocimiento de su disciplina, o para criticar los aportes realizados por otros autores. Por su parte, la dimensión social recoge aquellas estrategias que el autor utiliza para valorarse positivamente a sí mismo, mostrándose como un miembro legítimo de su comunidad y destacando sus características positivas como investigador. Ello puede realizarse mediante la autovaloración positiva del autor o a través de la presentación de los errores o falencias de otros investigadores. Finalmente, la dimensión textual engloba las estrategias mediante las cuales el autor valora positivamente su texto o partes de él (Meza, 2017a). Todas las estrategias que constituyen el modelo empíricamente fundado del posicionamiento estratégico del autor, junto a su definición y ejemplificación, se pueden encontrar en Meza (2017b).

Particularmente, en esta investigación se propone como objetivo general analizar la variación del posicionamiento estratégico del autor en AIC, considerando dos variables: el área de la ciencia (Ciencias Sociales, Humanidades e Ingeniería y Tecnología) y los grados de experticia del autor en el ámbito de las publicaciones científicas (novatos y expertos). Un autor novato o en formación es aquel que publica por primera vez un AIC en una revista indexada, que son las mejor valoradas para acreditar investigación en Chile; mientras que un experto o consolidado es el que cuenta con más de una publicación del mismo tipo. La elección de las áreas comparadas obedece a que, tradicionalmente, se ha afirmado que el AIC es el principal instrumento para la difusión del conocimiento en Ciencias Naturales, pero no en Ciencias Sociales y Humanidades, donde la difusión se realiza principalmente a través de monografías no indexadas (Larivière et al., 2006); y tampoco en Ingeniería, donde las comprensiones teóricas se traducen, preferentemente, en aplicaciones (Gibbons et al., 1997). En síntesis, se incluyen áreas, que comparten el hecho de no utilizar los AIC en revistas indexadas como instrumento principal para la difusión del conocimiento. 
Es importante señalar que, si bien existen algunos trabajos que indirectamente entregan sugerencias a los investigadores interesados en publicar, ellas dicen relación, por ejemplo, con el tipo de revista recomendado (de acceso abierto) (Bongiovani et al., 2012), o con las conductas inapropiadas de los autores (Matías- Guiu y García-Ramos, 2010). Así, hasta la fecha, no existen estudios que den cuenta de las estrategias discursivas específicas que utilizan los autores para publicar sus artículos y atraer la atención de sus lectores. Tampoco existen trabajos sobre el posicionamiento del autor en las áreas y grados de formación aquí explorados. Esta investigación, además, tiene el valor de que las estrategias han sido identificadas a partir del análisis mismo de los textos del corpus, esto implica que no se realiza un análisis top-down (Tognini-Bonelli, 2001), aplicando modelos preconcebidos para otros propósitos.

Para cumplir con el objetivo propuesto se desarrolla una investigación de tipo mixto (cualitativo- cuantitativo), a partir de una exploración inductiva- deductiva de un corpus representativo de una población mayor, constituido por 165 Artículos de Investigación de las áreas y niveles de experticia de nuestro interés. A fin de asegurar la confiabilidad de la investigación, se han llevado a cabo distintos procedimientos de validación, en distintas etapas y con la participación de investigadores chilenos y extranjeros. A continuación, se describe con mayor nivel de detalle los métodos utilizados en esta investigación; para luego dar cuenta de los principales resultados obtenidos, junto a su respectiva discusión; y, finalmente, se cierra el trabajo con las conclusiones más importantes derivadas del estudio realizado.

\section{MÉTODOS}

Se desarrolló una investigación mixta (cualitativa- cuantitativa). Ello supone que interesa la profundidad de análisis de los estudios cualitativos y que, además, los resultados son generalizables y representativos de la población, pues se ha recogido una muestra representativa de ella (Hernández et al., 2006).

\section{Objetivos}

El objetivo general es analizar la variación del uso de estrategias de posicionamiento en AIC, considerando dos variables: área de la ciencia (Ciencias Sociales, Humanidades e Ingeniería y Tecnología) y grado de experticia del autor en el ámbito de las publicaciones científicas (novatos y expertos). Para cumplir con este objetivo, primero, se identificaron y describieron las estrategias de posicionamiento en todos los textos de los estratos analizados para, luego, llevar a cabo un análisis comparativo, considerando las variables planteadas.

\section{Corpus}

Los textos analizados en esta investigación son parte de un corpus de análisis mayor, denominado CORAI ACEX (Corpus Representativo de Artículos de Investigación por Áreas de la Ciencia y Grados de Experticia), recolectado en el marco del Proyecto FONDECYT de Postdoctorado $N^{\circ} 3150056$. En términos generales, todos los textos cumplen con las siguientes características: son AIC escritos en español por un autor único, publicados en Scielo Chile entre los años 2010 y 2014. En Meza (2017c) se puede encontrar una explicación detallada sobre las características de CORAI ACEX, y también sobre los procedimientos seguidos para su conformación. Para esta investigación, se seleccionaron los textos de autores novatos y expertos, pertenecientes a tres áreas: Ciencias Sociales, Humanidades e Ingeniería y Tecnología.

Cabe señalar que el corpus conformado obedece al cálculo de una muestra representativa de una población mayor (2482 artículos). Más específicamente, se ha aplicado un muestreo estratificado con afijación proporcional, lo que significa que el número de elementos muestrales de cada estrato es directamente proporcional al tamaño del estrato dentro de la población. Esto permite que todos los estratos de nuestro interés estén correctamente representados en la muestra (Martínez y Martínez, 2008). El nivel de precisión calculado fue de $7 \%$. Además, para seleccionar la muestra aleatoria de cada estrato se utilizó un generador de números aleatorios sin repetición (específicamente, el programa $R$, un software libre para cálculos estadísticos). Luego de la aplicación de todos los procedimientos mencionados, el corpus de esta investigación quedó constituido por 165 AIC, distribuidos de la forma mostrada en la Tabla 1.

Tabla 1: Conformación del corpus

\begin{tabular}{|l|c|c|c|}
\hline \multirow{2}{*}{\multicolumn{1}{|c|}{ Área De La Ciencia }} & \multicolumn{2}{|c|}{ Grado De Experticia } & \multirow{2}{*}{ Total } \\
\cline { 2 - 3 } & Novatos & Expertos & \\
\hline Ciencias Sociales & 67 & 20 & 87 \\
\hline Humanidades & 59 & 17 & 76 \\
\hline Ingeniería y Tecnología & 2 & 0 & 2 \\
\hline Total & 128 & 37 & 165 \\
\hline
\end{tabular}


Como se observa en la Tabla 1, tras la aplicación de los criterios de conformación del corpus y de la muestra, no se incluyen textos de autores expertos de Ingeniería y Tecnología. Ello se debe a que no se encontraron, en el período establecido, AIC en español escritos por un solo autor consolidado. Este hecho sugiere que, a mayor grado de experticia, los autores de Ingeniería y Tecnología privilegian el trabajo colaborativo por sobre el individual. De hecho, es posible advertir, a partir de la Tabla 1, que los autores novatos de Ingeniería también evidencian, en alguna medida, una tendencia a trabajar colaborativamente, pues en la muestra hay solamente dos textos escritos por autores únicos.

CORAI ACEX ha sido dividido entre un corpus de prueba, constituido por el $33 \%$ de los textos de cada estrato, y un corpus de investigación, conformado por el $67 \%$ de los textos restantes de cada grupo. Se ha incluido este procedimiento como un primer mecanismo de validación del análisis. Se considera necesaria esta partición, puesto que, dado que las categorías de análisis han surgido a partir del análisis de los textos, necesitan ser sometidas a una etapa de evaluación, que permita ajustar y precisar las categorías levantadas, esto es, incorporar, especificar, sintetizar, eliminar o incluir algunas otras. Así, tras el análisis del corpus de prueba, se realizaron los ajustes pertinentes y, luego, una vez que se aprobaron todos ellos, se prosiguió con el análisis del corpus de investigación.

\section{Métodos de obtención de datos}

Para realizar el análisis de los textos del corpus, y de manera similar a la investigación mayor en la que se enmarca este trabajo, el análisis se realizó bajo un enfoque ascendente- descendente (bottom up- top down) o inductivo- deductivo. Esto, porque, al momento de llevar a cabo el análisis de los textos del corpus, no se aplicó un modelo preexistente, sino que, siguiendo los parámetros de dicho enfoque, se adoptó una postura abierta y, en cierto modo, inocente frente a los textos que son objeto de análisis, de modo que sean los textos mismos quienes guíen la obtención de las categorías de análisis. Al mismo tiempo, la responsable de la investigación lleva consigo conocimientos previos que no puede dejar de lado, por lo que, en este sentido, no es un estudio puramente inductivo. Esta metodología es respaldada por Tognini- Bonelli (2001), cuando afirma que "it is important to understand here that this methodology is not mechanical, but mediated constantly by the linguist, who is still behaving as a linguist and applying his or her knowledge and experience and intelligence at every stage during this process. There is no such a thing as pure induction" (Tognini-Bonelli, $2001: 85)$. Sin duda, esta mirada complementaria y no excluyente permite tener una visión más completa del fenómeno del posicionamiento estratégico del autor en Artículos de Investigación.

\section{Procedimientos de validación del análisis}

Además de la división entre el corpus de prueba y el de investigación, el análisis se sometió a diversos procedimientos de validación, de modo de asegurar que el levantamiento de las categorías y el etiquetaje de los textos no respondan a una impresión subjetiva de la investigadora responsable. Estos procedimientos de validación fueron aplicados de manera independiente al corpus de prueba y al corpus de investigación, y considerando el total de textos de CORAI ACEX. Los procedimientos específicos de validación son: triangulación, revisión por parte de expertos y juicio de evaluadores expertos. Cada uno de ellos se describe detalladamente a continuación. En primer lugar, en cuanto a la triangulación, una vez que el análisis de la investigadora responsable arrojó las categorías de posicionamiento, se ejercitó a 3 asistentes de investigación para que, con dichas categorías, realizaran el análisis de algunos AIC del corpus, a fin de corroborar que el análisis no fuera subjetivo. A las ayudantes se les solicitó que registraran cada uno de los casos con los que no estaban de acuerdo o que les producían dudas. Luego, dichos casos fueron revisados en conjunto con la investigadora responsable hasta que se eliminó cada duda.

En segundo lugar, para la revisión por parte de expertos, se realizó una revisión de las categorías de análisis obtenidas, así como también del método utilizado para recogerlas, mediante la consulta a dos investigadores (uno chileno y uno extranjero), ambos doctores en lingüística, hablantes nativos de español, expertos en escritura académica y científica. Todas las dudas y observaciones entregadas por ellos se resolvieron con la investigadora responsable, incorporando todas las sugerencias pertinentes.

Por último, en cuanto al juicio de evaluadores expertos, su objetivo central fue evaluar la confiabilidad del modelo obtenido, constituido por todas las categorías de posicionamiento identificadas tras el análisis, primero, del corpus de prueba y, luego, del corpus de investigación. La confiabilidad fue obtenida a partir del grado de acuerdo existente entre un grupo de evaluadores expertos sobre las categorías de análisis de posicionamiento. Para solicitar la evaluación a los jueces, se creó un documento en el que se detalló el objetivo de la investigación y algunos aspectos centrales de la misma (corpus, procedimientos de análisis, etc.). Se expuso detalladamente cada una de las categorías a evaluar, incorporando el nombre de la categoría, su descripción y ejemplificación. Además, se creó una escala Likert de 3 niveles ("Acepta", "Corrige" o "Rechaza"). Se optó por este tipo de herramienta de evaluación por las diversas ventajas que se han 
registrado, entre las que destaca que resulta muy fácil convertir los datos a una escala de medida para, luego, poder aplicar algún tipo de análisis estadístico (Jackson, 2009) y obtener así el grado y porcentaje de acuerdo entre jueces. Así, dada la gran cantidad de datos a evaluar, la Escala Likert resulta óptima para los propósitos de esta investigación. Se agregó, además, un espacio para que los jueces pudieran incorporar todas las observaciones cualitativas que consideraran pertinentes.

Los evaluadores seleccionados, tanto para la validación del 33\% como del 100\%, son todos doctores o doctoras en lingüística, hablantes nativos de español, especialistas en escritura académica o científica, chilenos y extranjeros. Para la validación del corpus de prueba y del corpus de investigación se recurrió a 3 expertos en cada ocasión. En ambas etapas, se calculó el porcentaje de acuerdo entre jueces y, además, se realizó un análisis estadístico del acuerdo, utilizando el coeficiente Kappa de Fleiss (1971), cuyo objetivo es determinar el grado de acuerdo entre tres o más evaluadores mediante la exclusión de las concordancias que se puedan atribuir al azar (Fleiss, 1971). Tanto el porcentaje de acuerdo como el coeficiente Kappa de Fleiss (1971) fueron calculados con la herramienta online ReCal3: Reliability for 3+ Coders, disponible de forma gratuita en http://dfreelon.org/utils/recalfront/recal3/.

Los resultados obtenidos tras el cálculo del porcentaje de acuerdo y del coeficiente Kappa de Fleiss (1971) son los que se detallan a continuación. Primero, en cuanto al porcentaje de acuerdo, para el corpus de prueba obtuvimos un $66,67 \%$, mientras que para el de investigación el porcentaje subió considerablemente a un $90,48 \%$. Al respecto, cabe agregar que la bibliografía indica que, en los procesos de validación, se debe tomar como valor de referencia mínimo un 70\% (Blessing y Chakrabarti, 2009). En consecuencia, si bien tras el análisis del corpus de prueba el análisis no cumplió con este porcentaje mínimo, cualquier inconveniente fue resuelto, pues para el corpus de investigación se obtuvo un valor que supera ampliamente el mínimo establecido. Ahora bien, en cuanto al coeficiente Kappa de Fleiss (1971), para el corpus de prueba se alcanzó un valor de 0,67, número que Landis y Koch (1977) interpretan como un acuerdo moderado. Luego, para el corpus de investigación, se logró un coeficiente igual a 0,91, que, según los mismos autores, corresponde a un acuerdo casi perfecto, que es la categoría más alta que se podría alcanzar. Ambos análisis estadísticos evidencian, entonces, la importancia que tiene el hecho de realizar el análisis, parcelando los textos entre un corpus de prueba y uno de investigación.

\section{RESULTADOS Y DISCUSIÓN}

En este punto, se da cuenta de los resultados obtenidos tras la aplicación de todos los procedimientos metodológicos antes detallados.

\section{Tipos de estrategias}

Como se señaló en la introducción de este trabajo, las estrategias de posicionamiento del autor se agrupan en tres dimensiones: epistémica, social y textual. A continuación, en la Figura 1, se presenta la proporción con la que aparecen dichas dimensiones en el corpus.

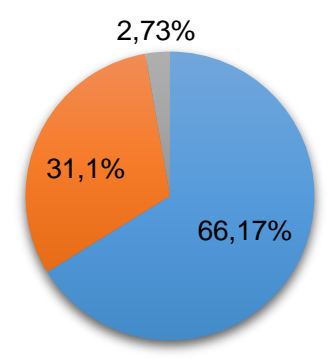

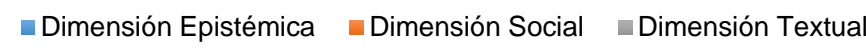

Fig. 1: Proporción de tipos de estrategias de posicionamiento

En el corpus analizado se identificó un total de 2954 estrategias de posicionamiento del autor. Como se advierte a partir de la información presentada en la Figura 1, la mayor proporción corresponde a las estrategias de la dimensión epistémica (66,17\%). Ello implica que, si bien los autores intentan posicionar su trabajo mediante la inclusión de diversos tipos de estrategias en sus textos, en términos generales, prima ampliamente el interés por evidenciar el aporte que se hará al conocimiento con el trabajo, por sobre la valoración que pueda hacer de sí mismo como investigador mediante las estrategias sociales (31,1\%), que es la segunda preocupación más frecuente de los autores; o de las características textuales de su trabajo a través de la dimensión textual (2,73\%), que ocupa el tercer lugar de preferencia. A continuación, se presentan 
ejemplos de casos de estas tres dimensiones. Antes de ello, es importante señalar que la información que aparece entre corchetes al final de cada ejemplo corresponde al código que identifica al texto en el corpus.

a) Dimensión epistémica: se presenta un caso asociado a la estrategia Destacar el aporte o la utilidad de su investigación, mediante la cual el investigador destaca el aporte al conocimiento que hará con su estudio. Por ejemplo: "Los resultados de esta investigación completan el espacio vacío entre el conocimiento de edificabilidad y la medida de los factores que impactan la operación de moldeado de las vigas rasantes, que pueden ser usados como retroalimentación a los diseñadores sobre cuán bien sus diseños consideran los requerimientos de principios de edificabilidad, y las consecuencias de sus decisiones sobre productividad laboral" [IYT_F_2].

b) Dimensión social: El ejemplo presentado corresponde a la estrategia Manifestar excelencia de trabajos propios, a través de la cual el autor manifiesta la excelente calidad que poseen sus investigaciones previas. El caso específico es: "Este artículo constituye una versión revisada de la parte general de la tesis de magíster, que fuera evaluada con máxima distinción" [CS_F_56].

c) Dimensión textual: El caso expuesto pertenece, específicamente, a la estrategia Valorar positivamente la organización de su texto, mediante la cual el autor valora positivamente la organización que ha hecho de su texto. Por ejemplo: "La organización de la literatura bajo cinco encabezamientos que propone este artículo ofrece un punto de partida para una discusión acerca de las diferencias y similitudes entre las múltiples explicaciones que se han propuesto" [CS_C_788].

Ahora bien, si se considera la presencia de las distintas dimensiones desagregadas de acuerdo con las diferentes áreas de la ciencia y grados de experticia aquí estudiados, los resultados son los que se presentan, a continuación, en la Figura 2:

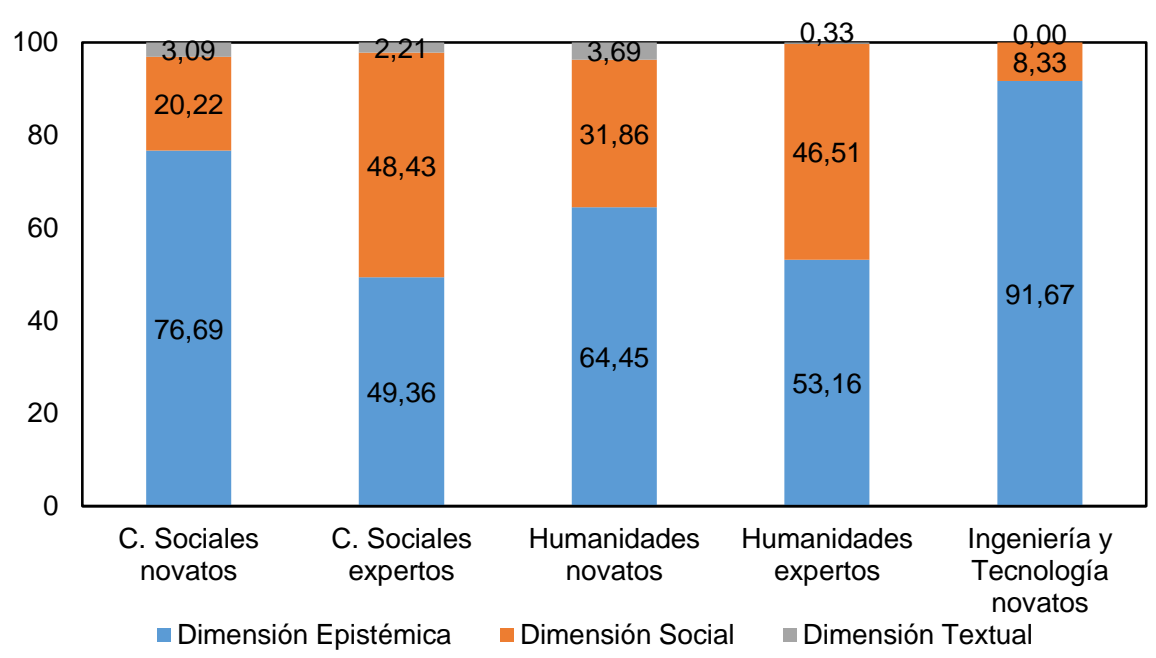

Fig. 2: Proporción de tipos de estrategias de posicionamiento por área de la ciencia y grado de experticia

Sobre la base de la información presentada en la Figura 2, se puede advertir que las tres dimensiones (epistémica, social y textual) están presentes en todas las áreas y grados de experticia estudiados, a excepción de los autores de Ingeniería y Tecnología en formación, estrato en el que no se encontraron estrategias asociadas a la dimensión textual. Ello demuestra que los autores que se están formando en dicha área de la ciencia privilegian ampliamente la dimensión epistémica para posicionar sus investigaciones $(91,67 \%)$, centrándose, principalmente, en el aporte al conocimiento que harán con su trabajo; además, son autores que recurren muy escasamente a las estrategias de la dimensión social (8,33\%); y, por último, no consideran como una vía posible de posicionamiento el hecho de destacar los atributos positivos de sus textos, pues prescinden de la dimensión textual. Por tanto, es posible afirmar que las estrategias epistémicas y, en menor medida, las sociales serían transversales a las distintas áreas de la ciencia y grados de formación considerados en esta investigación, no así las de la dimensión textual que son exclusivas de Humanidades y Ciencias Sociales.

Así mismo, en todos los estratos analizados, el primer lugar de preferencia lo ocupa la dimensión epistémica, el segundo la dimensión social y el tercero la textual. En todos los casos predominaría, entonces, este deseo por posicionar los aportes que hará la investigación a un campo de conocimiento específico. Un caso especial es el de los textos de Ciencias Sociales producidos por autores consolidados, en cuyos textos la dimensión epistémica y social tienen casi la misma ocurrencia: $49,36 \%$ y $48,43 \%$, respectivamente. Ello sugiere que para dichos autores tiene, prácticamente, la misma importancia el posicionamiento del aporte de la investigación 
como de las características positivas del investigador. Un caso similar es el de los autores consolidados de Humanidades, aunque la distancia no es tan estrecha (53,16\% versus $46,51 \%)$. Por otro lado, es posible afirmar que el grupo que mayor proporción de estrategias epistémicas utiliza es Ingeniería y Tecnología en formación; en el caso de las estrategias sociales, quienes tienen mayor predilección por ellas son los autores consolidados de Humanidades; mientras que la mayor tendencia a posicionar aspectos textuales se la adjudican los autores novatos de Humanidades.

\section{Variedad de estrategias}

En este punto se muestra la variedad de estrategias de posicionamiento utilizadas en los distintos grupos analizados en esta investigación, esto es, cuántas estrategias distintas del total disponible se utilizan en los estratos estudiados. Estos datos se sistematizan, a continuación, en la Figura 3:

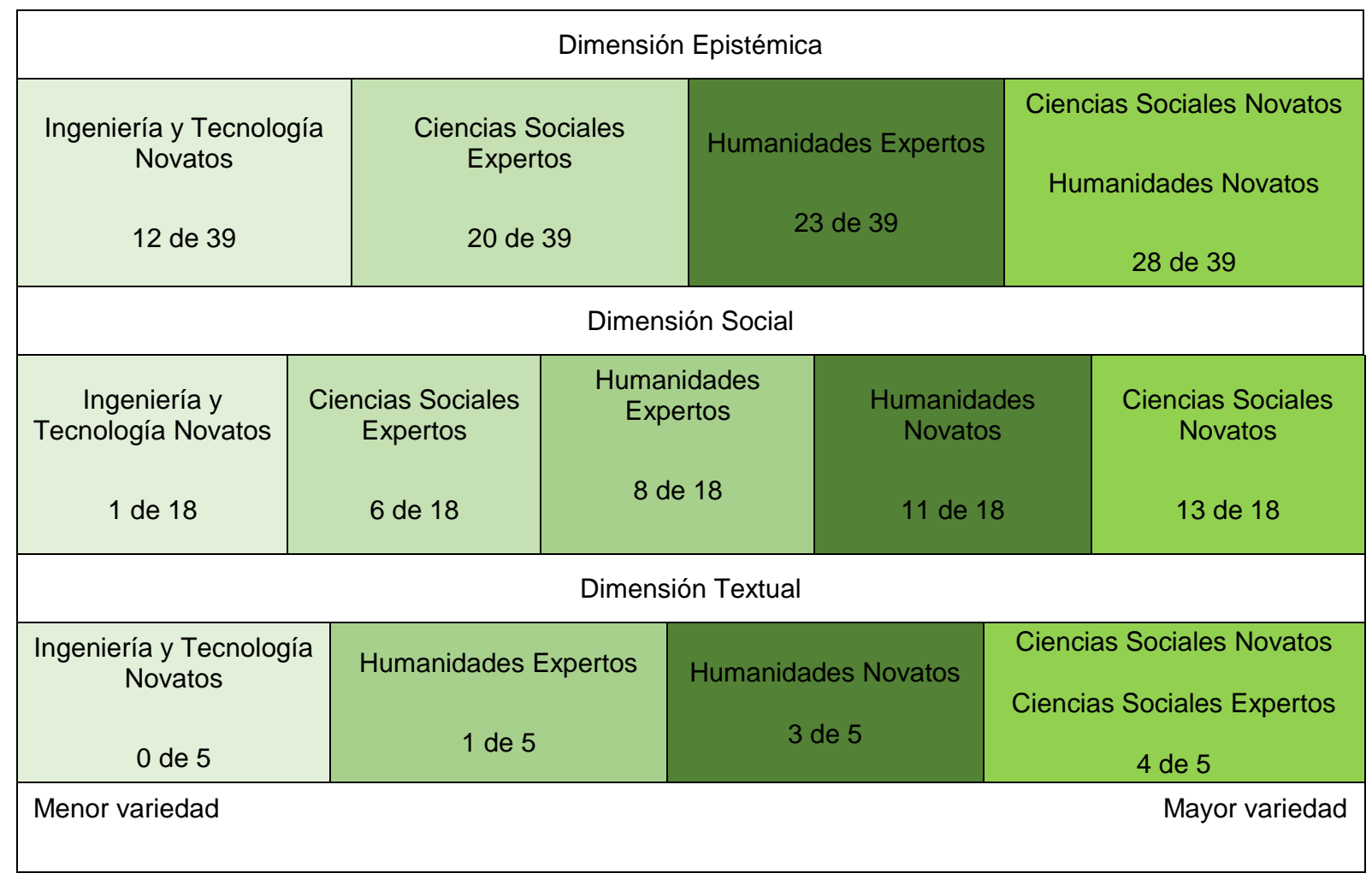

Fig. 3: Variedad de estrategias de posicionamiento

Como se observa en la Figura 3, en ninguno de los grupos analizados se utiliza el total de las estrategias identificadas en cada dimensión, sino que, en todos los casos, se utiliza una proporción de ellas, que varía según el grado de experticia y el área de la ciencia de los autores. Así, los escritores novatos de Ciencias Sociales son quienes, en todas las dimensiones, utilizan mayor variedad de estrategias de posicionamiento del Autor; mientras que, en el polo contrario, los escritores novatos de Ingeniería y Tecnología son quienes utilizan menor variedad de estrategias de las tres dimensiones que constituyen nuestra propuesta. En el caso de las estrategias epistémicas, el primer lugar es compartido por los autores de Ciencias Sociales y Humanidades en formación, quienes recurren a 28 de las 39 estrategias identificadas, aunque no corresponden exactamente a los mismos casos; y, en la dimensión textual, el primer lugar es para los autores novatos y expertos de Ciencias Sociales.

Si se considera la Figura 3 como una especie de continuum, un caso que llama la atención es el de los autores de Ciencias Sociales, pues, en las dimensiones epistémica y social, los escritores novatos se ubican en el extremo de mayor variedad de uso, mientras que los expertos se encuentran hacia el lado de menor variedad. Ello sugiere que, a medida que los autores de dicha disciplina se van especializando, van prescindiendo de algunos tipos de estrategias epistémicas y sociales para posicionar sus investigaciones. El caso de la dimensión textual es diferente, pues tanto los autores en formación como los consolidados de dicha área de la ciencia utilizan gran variedad de estrategias de la dimensión textual, ocupando el primer lugar de mayor variedad de uso. Por otro lado, es posible afirmar que Humanidades es un área más o menos estable, pues siempre se ubica en el punto central del continuum, no existiendo gran diferencia entre la variedad de uso de los autores novatos y expertos de la disciplina, aunque, al igual que en el caso de Ciencias Sociales, advertimos en todas las dimensiones una preponderancia de los autores en formación por sobre los consolidados en la variedad de uso de estrategias de posicionamiento. 


\section{Cantidad de estrategias}

En este punto se muestran los resultados referentes a la cantidad de estrategias de posicionamiento, esto es, cuántas estrategias han sido identificadas en cada estrato analizado. De acuerdo con su frecuencia de aparición, las 2.954 estrategias registradas en CORAI ACEX se distribuyen en los distintos grupos estudiados, tal como se muestra en la Figura 4.
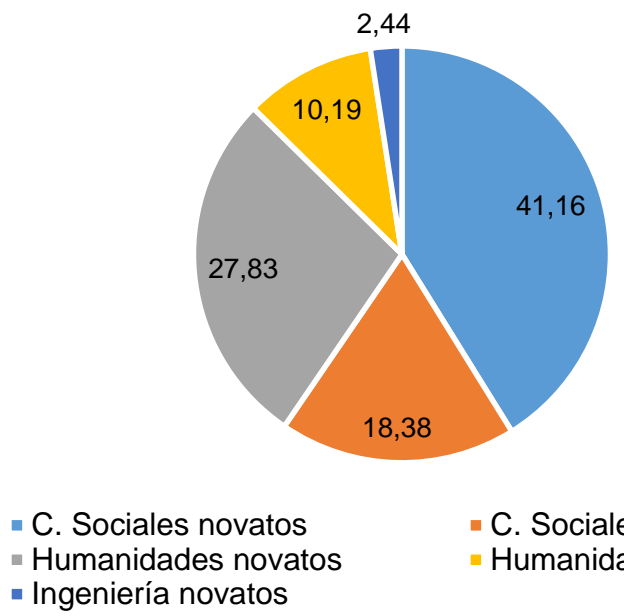

- C. Sociales expertos

- Humanidades expertos

- Ingeniería novatos

Fig. 4: Frecuencia de uso de estrategias de posicionamiento del Autor

Como se observa en la Figura 4, son los autores novatos de Ciencias Sociales, quienes utilizan mayor cantidad de estrategias de posicionamiento, alcanzando más del $40 \%$ del total. De hecho, si se considera Ciencias Sociales como un solo grupo, esto es, autores novatos y expertos, es posible advertir que es esta área la que ocupa más de la mitad del total de estrategias identificadas (59,54\%). El segundo lugar de mayor frecuencia de uso es para los autores en formación de Humanidades, el tercero para los consolidados de la misma área, y el último se lo llevan los novatos de Ingeniería y Tecnología.

Dicha distribución de los datos evidencia que el mayor uso de estrategias de posicionamiento está directamente relacionado con la necesidad de los autores por ganarse un espacio en la comunidad de investigadores. Así, por una parte, son los autores de Ciencias Sociales y Humanidades quienes lideran el uso de estrategias de posicionamiento porque son ellos, precisamente, quienes han tenido que lidiar por tener un lugar en el campo de la investigación, esto es, reconocimiento y financiamiento.

Del mismo modo, se observa que son los autores novatos de dichas áreas quienes utilizan mayor cantidad de estrategias, pues son quienes tienen que demostrar en su comunidad que son capaces de aportar al conocimiento, lo que evidencian a través de estrategias de la dimensión epistémica; que son sujetos valiosos con las características idóneas para pertenecer a cierta comunidad, lo que revelan mediante la dimensión social; y que escriben textos bien preparados, lo que manifiestan con las estrategias textuales. En esta misma línea, la baja frecuencia de uso de estrategias de posicionamiento por parte de autores de Ingeniería se debe a que, en su caso, la importancia de la investigación está acreditada por la reputación que posee la disciplina en el ámbito científico, en particular, y en la sociedad, en general.

Ahora bien, para dar cuenta más detalladamente de la cantidad de estrategias que utilizan los autores en los distintos estratos, se presentan tres tablas, una por cada dimensión. Todas ellas responden a los mismos criterios de organización, ya validados en Meza (2015), y que se detallan a continuación. Se han dejado en blanco todas aquellas celdas correspondientes a estrategias ausentes en los estratos analizados. Por su parte, las estrategias que sí aparecen se han clasificado en tres grupos de acuerdo con su frecuencia al interior del estrato, identificando cada grupo con un color distinto: rojo para las estrategias de alta frecuencia, amarillo para las de ocurrencia media y azul para las de baja frecuencia.

Para realizar esta división, se ha considerado el valor mayor de cada estrato y se ha dividido por tres, resultado que se ha utilizado para establecer los tres rangos. Por ejemplo, en la dimensión epistémica, si se toma el caso de Ciencias Naturales en formación, su valor de ocurrencia más alto es 20,37\%. Ese número se ha dividido por tres, pues se establecieron tres rangos (alta, media y baja frecuencia). Así, en dicho grupo, las estrategias de baja frecuencia serían aquellas cuyo valor fuera entre 0,01 y 6,79, las de frecuencia media entre 6,8 y 13,58 y las de frecuencia alta las que se ubicaran sobre $13,59 \%$ de ocurrencia. En la Tabla 2 , se presentan los resultados para la dimensión epistémica: 
Tabla 2: Cantidad de estrategias de la dimensión epistémica. Leyenda: CS_N: Ciencias Sociales novatos, CS_E: Ciencias Sociales expertos, HUM_N: Humanidades novatos, HUM_E: Humanidades expertos, IYT_N: Ingeniería y Tecnología novatos

\begin{tabular}{|c|c|c|c|c|c|}
\hline & $C S \_N$ & CS_E & HUM_N & $\begin{array}{l}H_{E}^{H U M} \\
-\end{array}$ & $I Y T_{-} N$ \\
\hline 1 Validar resultados propios CAR & 0,22 & & & & \\
\hline 2 Justificar propuesta de investigación u objeto de estudio CAR & 0,54 & & & & \\
\hline 3 Atribuirse avance en su área & & 0,37 & & & \\
\hline 4 Destacar otras aplicaciones de su investigación & & & 0,19 & & \\
\hline 5 Expresar postura propia CAR & & & 0,57 & & \\
\hline 6 Recomendar procedimiento CAR & & & 0,19 & & \\
\hline 7 Explicitar que presenta información inédita & & & & 1,25 & \\
\hline $\begin{array}{l}8 \text { Destacar carácter único de su investigación o de algún aspecto } \\
\text { de ella }\end{array}$ & & & & 1,25 & \\
\hline 9 Destacar que la investigación propia es mejor CAR & & & & 0,63 & \\
\hline $\begin{array}{l}10 \text { Declarar la dificultad de su investigación o su objeto de estudio } \\
\text { CAR }\end{array}$ & 0,54 & & 0,38 & & \\
\hline 11 Confirmar personalmente la información entregada & 0,22 & & & 0,63 & \\
\hline 12 Justificar decisión teórica o metodológica CAR & 0,11 & & 0,19 & & \\
\hline 13 Señalar que ha mejorado el método utilizado & 0,87 & & 0,38 & & \\
\hline $\begin{array}{l}14 \text { Destacar la importancia de su área o de su objeto de estudio } \\
\text { CAR }\end{array}$ & 0,22 & & 0,38 & 0,63 & \\
\hline 15 Validar resultados propios & 3,38 & & 0,76 & & 3,03 \\
\hline 16 Introducir un concepto & 0,33 & 0,75 & 1,15 & & \\
\hline 17 Destacar que la investigación propia es mejor & 1,09 & 0,37 & 0,57 & & \\
\hline 18 Mostrar acuerdo con otros autores y teorías & 4,79 & 1,87 & 4,96 & 5,63 & \\
\hline 19 Entregar definición o explicación propia para un concepto & $\overline{0,44}$ & 1,12 & 0,76 & 1,88 & \\
\hline 20 Declarar la dificultad de su investigación o su objeto de estudio & 1,09 & 3,73 & 2,48 & 1,25 & \\
\hline 21 Justificar decisión teórica o metodológica & 1,63 & 0,75 & 0,95 & 0,63 & \\
\hline 22 Justificar limitaciones & 6,97 & 7,46 & 7,06 & 1,25 & \\
\hline 23 Realizar proyección o predicción & 0,33 & 2,24 & 1,15 & 1,88 & \\
\hline 24 Justificar propuesta de investigación u objeto de estudio & $\overline{3,16}$ & 4,48 & 2,29 & 3,75 & \\
\hline 25 Valorar positivamente su diseño o método de investigación CAR & 0,44 & & 0,57 & 0,63 & 1,52 \\
\hline 26 Destacar el aporte o la utilidad de su investigación & 5,99 & 3,36 & 9,35 & 11,88 & 24,24 \\
\hline 27 Criticar investigaciones ajenas o un aspecto de ellas & 13,29 & 15,30 & 19,85 & 11,88 & 18,18 \\
\hline 28 Criticar investigaciones ajenas o un aspecto de ellas CAR & 0,33 & 0,37 & 1,15 & 3,13 & 3,03 \\
\hline 29 Destacar la importancia de su área o de su objeto de estudio & 5,01 & 5,60 & 4,20 & 3,13 & 7,58 \\
\hline 30 Expresar postura propia & 11,11 & 21,27 & 18,13 & 17,50 & 4,55 \\
\hline 31 Presentar propuesta de investigación & 8,71 & 10,07 & 8,59 & 10,63 & 4,55 \\
\hline 32 Recomendar procedimiento & 2,18 & 0,75 & 1,72 & 0,63 & 3,03 \\
\hline 33 Identificar vacío, problema no resuelto o controversia CAR & 4,68 & 1,87 & 2,29 & 3,13 & 12,12 \\
\hline 34 Identificar vacío, problema no resuelto o controversia & 20,37 & 17,54 & 8,59 & 13,13 & 15,15 \\
\hline 35 Valorar positivamente su diseño o método de investigación & 1,96 & 0,75 & 1,15 & 3,75 & 3,03 \\
\hline
\end{tabular}

Antes de comentar los resultados, es importante señalar que, en el modelo general de análisis, algunas de las estrategias tienen una especie de duplicación, pues cuentan con una segunda versión que se diferencia solo porque son presentadas acompañadas de referencias de otras investigaciones. Es el caso, por ejemplo, de "Criticar investigaciones ajenas o un aspecto de ellas", estrategia mediante la cual el investigador realiza críticas a otros trabajos; y que encuentra su duplicación en "Criticar investigaciones ajenas o un aspecto de ellas con apoyo de referencias de otros autores", a través de la que se realiza una crítica, pero se respalda en lo que han dicho otros autores, otorgándole, así, mayor fundamento a sus críticas. Por razones de espacio, todas aquellas estrategias que cuenten con este apoyo de referencias se han identificado con la sigla "CAR" ("con apoyo de referencias de otros autores"). Esto es válido para las dimensiones epistémica y social, pues en la dimensión textual no se registraron estrategias de este tipo. 
Ahora bien, en cuanto a los resultados mismos, es posible observar que existen estrategias exclusivas de algunos estratos, otras compartidas entre algunos de ellos y otras transversales a todas las disciplinas y grados de experticia analizados. Las estrategias transversales son las que, en la tabla, se ubican entre el número 26 al 35, y que, en consecuencia, serían las estrategias centrales para posicionarse estratégicamente en cualquier área de la ciencia e independientemente del grado de experticia del autor. Entre dichas estrategias destacan los casos: Criticar investigaciones ajenas o un aspecto de ellas e Identificar vacío, problema no resuelto o controversia, pues ellas, en todos los estratos, aparecen con un nivel de ocurrencia alto o medio. Por tanto, la presentación de un vacío o problema no resuelto, así como también la crítica a otros trabajos serían las estrategias fundamentales en todos los grupos para llevar a cabo el posicionamiento estratégico del autor. Ejemplos de ambas estrategias son los siguientes: a) Identificar vacío, problema no resuelto o controversia: "A pesar de la importancia de este oficio para el concreto reforzado en obras, la influencia de los factores de edificabilidad sobre la productividad del oficio del moldeado sobre elementos estructurales mayores, aún está por ser cuantificada en términos medibles" [IYT_F_2]; b) Criticar investigaciones ajenas o un aspecto de ellas: "La manera como se ha construido esta supuesta limitación a la potestad invalidatoria es, a mi parecer, incorrecta" [CS_C_1828].

En el caso específico de la estrategia Identificar vacío, su alta ocurrencia se puede atribuir a que se trata de una estrategia transversal a la escritura académica y científica. La inclusión de esta categoría sería, entonces, una característica propia del género, mediante la cual el autor evidencia que existe un nicho en el conocimiento que se necesita cubrir con el Artículo presentado. De esta manera, la presentación del vacío se constituye en la mejor justificación para evidenciar la necesidad de llevar a cabo su investigación. En el corpus, esta función es de alta frecuencia en Ciencias Sociales (novatos y expertos) y en los autores consolidados de Humanidades; y es de ocurrencia media en los textos producidos por novatos de Ingeniería y Humanidades. Ello sugiere que, a mayor grado de experticia, mayor necesidad existe por explicitar los vacíos que vendrá a llenar la investigación presentada, lo que podría explicarse por un mayor conocimiento del género AIC. Ahora bien, a diferencia de otros trabajos, que restringen el estudio del vacío a la sección Introducción (Bunton, 2002), en el caso de esta investigación, se ha identificado dicha estrategia a lo largo de todo el texto.

Ahora bien, la importante presencia de la estrategia Criticar investigaciones ajenas o un aspecto de ellas en todos los grupos analizados evidencia que, independientemente del área de la ciencia a la que pertenezca el autor o de su grado de experticia en el ámbito de las publicaciones científicas, es una estrategia fundamental para posicionarse el hecho de dar cuenta de los aspectos negativos de investigaciones realizadas por otros autores. Así, los investigadores no solo recurren a la estrategia que implica mostrar vacíos para justificar sus trabajos, sino que también se valen de las características negativas de otros trabajos para demostrar la necesidad de realizar y publicar su investigación. El hecho de fundamentar la investigación acudiendo a una de estas dos estrategias, permite clasificar a los autores en dos grupos de acuerdo con el tipo de identidad autorial que proyectan. Así, existen autores constructivos, que son aquellos que utilizan más frecuentemente la indicación de un vacío como método de validación de la necesidad de su investigación o controversia; y autores correctores, que son quienes prefieren la crítica como método de validación de la necesidad de su investigación. En este sentido, serían más bien constructivos los autores de Ciencias Sociales (novatos y expertos) y los consolidados de Humanidades, mientras que los correctores serían los novatos de Humanidades e Ingeniería. Se puede afirmar, entonces, que la fundación de la investigación a partir de la crítica a otros es propia de autores que se están iniciando en la producción de publicaciones científicas. A juicio de la autora, el hecho de criticar otras investigaciones le permite al autor mostrarse como un sujeto que no solo es capaz de aportar a su comunidad con sus propias investigaciones, sino que también tiene la capacidad de evaluar el conocimiento producido en su comunidad y tomar una postura crítica al respecto, mostrándose, así, como un investigador con juicio propio, que no acepta fácilmente toda la información que se difunde en su área.

La estrategia epistémica más frecuente varía en los distintos estratos, aunque se registran algunas coincidencias. Este resultado es relevante, pues revela el ethos de cada grupo, en el sentido que evidencia cuál es la forma predilecta que tienen los autores para posicionar su investigación, resultado que refleja diferencias disciplinares y de experticia. Así, la estrategia más utilizada por los autores de Ciencias Sociales en formación es Identificar un vacío, problema no resuelto o controversia (20,37\%), por lo que estos autores mostrarían un ethos más bien propositivo al intentar cubrir los vacíos de conocimiento que persisten en su disciplina. Los autores expertos de dicha área, en cambio, tienen predilección por el uso de la estrategia Expresar postura propia $(21,27 \%)$, lo que da cuenta de un ethos de investigador opinante, que es capaz de manifestar su posición ante ciertos temas de interés de su área, como, por ejemplo: "El Proyecto, pese a despertar nuestra confusión en la contestación a la demanda, podemos decir que es justo si entendemos por justicia dar a todos los mismo" [CS_C_1006].

Los investigadores novatos de Humanidades utilizan más frecuentemente la estrategia Criticar investigaciones ajenas o un aspecto de ellas (19,85\%), por lo que, de acuerdo con lo señalado anteriormente, 
su perfil sería de autores correctores. Los escritores consolidados de esta misma área coinciden con el perfil de los autores expertos de Ciencias Sociales, pues utilizan preferentemente la estrategia Expresar postura propia $(17,5 \%)$, mostrándose como escritores opinantes. Finalmente, los investigadores novatos de Ingeniería y Tecnología utilizan mayormente la estrategia Destacar el aporte o la utilidad de su investigación $(24,24 \%)$, por lo que ostentan el ethos de contribuyente a su área de estudio, de un sujeto que participa en su comunidad disciplinar y que es capaz de contribuir a ella con su trabajo, tal como se evidencia en el siguiente caso: "Los resultados obtenidos, no sólo comprueban la importancia de aplicar principios de "racionalización" y "estandarización", en particular "modularidad", a la etapa de diseño de proyectos de construcción, facilitando el proceso de aprendizaje, permitiendo así a los operadores predecir problemas y racionalizar el proceso de solución de dificultades, evitar errores, compartir información, reducir material de desecho, por lo tanto aumenta la eficiencia y optimiza el gasto en la evolución de la edificación; sino que también probar el impacto positivo del concepto "economía de escala", el que además es incrementado por la aplicación de estos principios sobre la productividad de la operación de moldeado" [IYT_F_2]. En este sentido, los investigadores de Ingeniería y Tecnología serían los más estratégicos al momento de revelar su ethos investigativo. Los resultados de esta dimensión dan cuenta del perfil general de los autores, pues las estrategias epistémicas son las más utilizadas por todos los grupos.

Por otro lado, los resultados de la dimensión social son los que se presentan en la Tabla 3.

Tabla 3: Cantidad de estrategias de la dimensión social. Leyenda: CS_N: Ciencias Sociales novatos, CS_E: Ciencias Sociales expertos, HUM_N: Humanidades novatos, HUM_E: Humanidades expertos, IYT_N: Ingeniería y Tecnología novatos.

\begin{tabular}{|c|c|c|c|c|c|}
\hline & CS_N & CS_E & HUM_N & HUM_E & $I Y T_{-} N$ \\
\hline 1 Declarar ausencia de conflictos de interés & 1,65 & & & & \\
\hline 2 Declarar que la revista le solicita artículo & 0,41 & & & & \\
\hline 3 Declarar membresía & 0,41 & & & & \\
\hline 4 Explicitar orientación a otros & 0,41 & & & & \\
\hline $\begin{array}{l}5 \text { Destacar el prestigio o el aporte de la comunidad a la que } \\
\text { pertenece }\end{array}$ & & 0,76 & & & \\
\hline 6 Declarar especialidad & & & 0,39 & & \\
\hline $\begin{array}{l}7 \text { Destacar el prestigio o el aporte de la comunidad a la que } \\
\text { pertenece CAR }\end{array}$ & & & & 0,71 & \\
\hline 8 Manifestar excelencia de trabajos propios & 0,41 & & 0,77 & & \\
\hline 9 Corregir errores ajenos & & & 1,16 & 2,86 & \\
\hline 10 Evidenciar experiencia en investigación & 0,41 & & 0,77 & & \\
\hline 11 Explicitar traducción propia & 1,24 & & 0,77 & & \\
\hline 12 Mencionar grado académico en desarrollo & 0,83 & & 0,77 & 0,71 & \\
\hline 13 Recomendar bibliografía propia al lector & 4,96 & 10,65 & 8,11 & 6,43 & \\
\hline 14 Mencionar cargo & 7,02 & 4,56 & 3,47 & 5,00 & \\
\hline 15 Recomendar bibliografía al lector & 43,39 & 60,46 & 41,70 & 30,00 & \\
\hline 16 Mencionar título o grado académico & 10,74 & 1,90 & 3,09 & 1,43 & \\
\hline 17 Autorreferencia & 28,10 & 21,67 & 39,00 & 52,86 & 100 \\
\hline
\end{tabular}

Antes de describir los resultados, es importante señalar que la inclusión de algunas de las estrategias de la dimensión social podría entenderse como respuesta a una exigencia editorial de presentación del autor, como, por ejemplo: declarar membresía, declarar especialidad, mencionar grado académico en desarrollo, mencionar cargo y mencionar título o grado académico. Sin embargo, es posible asegurar que efectivamente son estrategias de posicionamiento, puesto que en la investigación se han considerado solo aquellos casos en que la revista no exige dichos datos, esto es, cuando el autor los ha incluido motu proprio. Para ello, se han revisado todas las normas a los autores de las revistas en que aparecen los Artículos de nuestro corpus, y se han etiquetado solo aquellos datos que la revista no exige, pero que el autor incluye. Así, la mención de dicho tipo de información contribuye a mostrar que las afirmaciones presentadas están respaldadas por alguien importante, que posee cierto título o grado y que pertenece a algún grupo de prestigio.

En cuanto a los resultados mismos, en la Tabla 3 se observa que se presenta solo una estrategia transversal a todas las disciplinas y grados de experticia analizados, que es la Autorreferencia, vale decir, aquellos casos en que el autor usa como referencia sus propios trabajos. Ello evidencia que esta es una práctica transversal a las distintas áreas de la ciencia y grados de experticia, y, además, que se trata de una práctica frecuente, pues en todos los estratos es de ocurrencia media o alta. Un aspecto interesante con respecto al uso de la 
Autorreferencia en autores novatos es el hecho de que pareciera que ellos imitan una práctica de los investigadores expertos, quienes suelen autocitarse, pero como son novatos y no cuentan con publicaciones previas importantes, citan documentos que se pueden encasillar como literatura gris, esto es: tesis, ponencias, etc. Por ejemplo: "ELTIT, Verónica. Impacto de la globalización en ciudades intermedias: el caso de Temuco, Región de la Araucanía. Universidad Autónoma de Chile. 2003. Seminario para optar al grado de licenciado en Arquitectura". Así, aparentemente, los autores en formación imitan prácticas que han visto en los consolidados, pero utilizando las herramientas de las que disponen.

En esta misma línea, como se puede apreciar en la Tabla 3, hay dos categorías que podrían asociarse a la práctica de la autocita, una es la Autorreferencia, explicada anteriormente, y, la otra, Recomendar bibliografía propia al lector, que refiere a aquellos casos en que el autor recomienda al lector que revise sus propios escritos. La diferencia entre ambas radica en que a través de la primera estrategia el autor presenta una referencia propia para sustentar ciertas afirmaciones; la segunda, en cambio, el escritor directamente sugiere revisar sus propios trabajos, los que, en ocasiones, ni siquiera tienen relación directa con el tema que se está tratando en el artículo. Un ejemplo de la segunda estrategia mencionada es: "Sobre la apropiación expresionista en Valparaíso ver mi trabajo: "La otra vanguardia: el expresionismo de Valparaíso en su revista Litoral"' [Hum_C_245].

Otra estrategia de frecuencia alta y media en todos los grupos analizados, excepto en Ingeniería y Tecnología donde no aparece, es Recomendar bibliografía al lector, que, de hecho, es la estrategia social más utilizada en tres de los cinco estratos analizados: Ciencias Sociales (novatos y expertos) y Humanidades en formación. A través de ella, el autor muestra al lector el dominio bibliográfico que posee. Un ejemplo de esta estrategia es la siguiente: "Pueden verse, al respecto, tanto el informe How Regions Grow de la OECD (2009) como el del Banco Mundial, Reshaping Economic Geography (The World Bank, 2009) o lo que se recoge en European Commission (2009)" [CS_F_1161]. En síntesis, entonces, las dos estrategias sociales más frecuentes dicen relación con la recomendación de literatura a los lectores, ya sea propia (Autorreferencia) o de otros (Recomendar bibliografía al lector). De esta manera, lo que más hacen los autores a través de las estrategias sociales es mostrarse como investigadores con dominio amplio de los temas tratados en su área, pues han publicado sobre dichos temas o porque son capaces de recomendar a los lectores referencias relacionadas con algún tema.

Una estrategia propia de los autores expertos que aquí analizamos es Destacar el prestigio o el aporte de la comunidad a la que pertenece, utilizada solo por los expertos de Ciencias Sociales, mientras que su variante CAR es empleada exclusivamente por el mismo tipo de autores de Humanidades. Esta es una estrategia que, como ya se ha señalado (Meza, 2017d), podría quedar fuera del posicionamiento del autor porque se posiciona a otros y no a sí mismo. Sin embargo, al destacar aspectos positivos de la comunidad de pertenencia, por extensión, se destaca a sí mismo al ser parte de esa comunidad. Esta estrategia se puede concretizar con o sin apoyo de otros autores. Un ejemplo es: "Particularmente en Chile, la comprensión del discurso escrito ha llamado la atención de muchos investigadores. Ejemplo de ello es el fructífero trabajo realizado por los especialistas de la Pontificia Universidad Católica de Valparaíso y, más precisamente, de la Escuela Lingüística de Valparaíso (ELV), donde se han realizado descripciones cada vez más precisas de los desempeños de los lectores en diferentes contextos de lectura ..." [Hum_C_1923]. El hecho de que esta sea una estrategia exclusiva de autores consolidados sugiere que posicionarse a través de la comunidad de pertenencia es algo que se adquiere después de algún tiempo de participación en la comunidad y no cuando recién se ingresa o se quiere ingresar a ella.

Por otro lado, existen algunas categorías propias de autores en formación, específicamente de Humanidades y Ciencias Sociales, que evidencian la necesidad que sienten los investigadores por demostrar en sus trabajos que son sujetos capacitados y que pueden aportar a su disciplina. El uso de estas estrategias, a juicio de la autora, evidencia la necesidad que tienen los autores en formación de las áreas mencionadas por ganarse una reputación y convencer a los gatekeepers de que son buenos elementos. Esta necesidad no es tan imperiosa para los autores expertos, pues ellos ya tienen un prestigio ganado. Algunos ejemplos de este tipo de estrategias son:

a) Explicitar orientación a otros: El autor señala que con la información expuesta en su artículo ha podido aconsejar u orientar a otros colegas, como en el siguiente caso: "Varios colegas epistemólogos e investigadores, al momento de intercambiar las ideas que se sostuvieron en este artículo, me plantearon "pero yo no uso hipótesis en ese sentido, no me ubico en la lógica de verificación" a lo que yo respondía "si no utiliza hipótesis en ese sentido, pues utilice pregunta. Llámelo de otro modo" [CS_F_367].

b) Declarar especialidad: El autor declara que es especialista en algún ámbito del conocimiento, especialidad que se autoasigna, pues no posee, de acuerdo con nuestros registros, algún tipo de estudio formal en el que se declara experto. Así, por ejemplo, encontramos en el corpus una autora que se declara "Especialista en ensayo mexicano" [Hum_F_631]. 
c) Manifestar excelencia de trabajos propios: Un ejemplo de esta estrategia, cuyo nombre, creemos, no necesita mayor explicación, es "Este artículo constituye una versión revisada de la parte general de la tesis de magíster, que fuera evaluada con máxima distinción, y cuyo título original era "Kulturelle Wertvorstellungen im Bereich des Verbotsirrtums"' [CS_F_56].

d) Evidenciar experiencia en investigación: El autor explicita que posee experiencia en investigación, señalando sus trabajos previos, trabajos que, por cierto, no son AIC publicados en revistas indexadas, como se muestra en el siguiente caso: "En 1999 comenzamos el vínculo con diversos grupos de artistas circenses de la ciudad de Buenos Aires. A lo largo de estos años, desde una metodología de tipo etnográfica, fuimos abordando el fenómeno desde distintas investigaciones que dieron como resultado algunos materiales en formato de documentales audiovisuales, diversos artículos, una tesis de licenciatura, "La carcajada y el asombro...» (2005) y una tesis doctoral, «Cultura, jóvenes y políticas en disputa...» (2012) ..." [CS_F_1173].

El hecho de que estas estrategias, así como la gran mayoría de las categorías de la dimensión social, se presenten solo en Ciencias Sociales y Humanidades, y no en Ingeniería y Tecnología, sugiere que son los autores de las áreas de la ciencia, que tradicionalmente han tenido que luchar por tener un reconocimiento social, quienes más variedad y cantidad de estrategias sociales de posicionamiento deben utilizar.

Además, existe una estrategia social que es exclusiva de un área de la ciencia, a saber, "Corregir errores ajenos", que es privativa de Humanidades. Ello implica que solo en esta área, tanto autores novatos como expertos, consideran una estrategia de posicionamiento válida el hecho de mostrar sus cualidades de autor instruido corrigiendo los errores de otros. Este dato viene a reforzar lo que se señalaba más arriba con respecto a la identidad correctiva que proyectan los autores novatos de Humanidades, quienes no solo fundan, principalmente, su investigación sobre la base de la crítica que hacen de los trabajos de otros autores, como ocurre en la dimensión epistémica, sino que, además, utilizan sus artículos para mostrar los errores de otros y corregirlos. De esta manera, actúan como una especie de censores de su disciplina.

Sobre la base de la estrategia social predilecta, tal como se hizo con la dimensión epistémica, es posible establecer el ethos que revelan los autores desde la dimensión social. Así, se manifiestan dos tipos de perfiles: uno, que es el de los autores de Ciencias Sociales (novatos y expertos) y de Humanidades en formación, que privilegian el uso de la estrategia ya comentada "Recomendar bibliografía al lector"; y que, en consecuencia, proyectan un ethos de autor ilustrado, mostrando al lector el dominio bibliográfico que posee. Otro, es el de los autores expertos de Humanidades y novatos de Ingeniería y Tecnología, quienes optan preferentemente por la Autorreferencia, evidenciando, así, un ethos de autor contribuyente a la disciplina, pues cita los trabajos que ha realizado previamente, aunque ellos no correspondan necesariamente a Artículos de Investigación publicados en revistas indexadas. Finalmente, los resultados de la dimensión textual son los que se exponen en la Tabla 4.

Tabla 4: Cantidad de estrategias de la dimensión textual. Leyenda: CS_N: Ciencias Sociales novatos, CS_E: Ciencias Sociales expertos, HUM_N: Humanidades novatos, HUM_E: Humanidades expertos, IYT_N: Ingeniería y Tecnología novatos.

\begin{tabular}{|c|c|c|c|c|c|}
\hline & CS_N & $C S \_E$ & HUM_N & HUM_E & IYT_N \\
\hline 1 Destacar la importancia de las referencias & 5,41 & & & & \\
\hline 2 Valorar positivamente su artículo (texto) & & 8,33 & & & \\
\hline 3 Valorar positivamente la organización de su texto & 8,11 & 16,67 & 3,33 & & \\
\hline 4 Agradecer las revisiones del texto & 54,05 & 66,67 & 53,33 & & \\
\hline 5 Destacar que el artículo es parte de un trabajo mayor & 32,43 & 8,33 & 43,33 & 100 & \\
\hline
\end{tabular}

En la dimensión textual no se identificó ninguna estrategia transversal a todos los grupos analizados, debido a que los autores de Ingeniería y Tecnología no recurren a esta dimensión. Solo se encontró una estrategia común a todos los demás estratos analizados, a saber, "Destacar que el artículo es parte de un trabajo mayor". Se considera este caso como una estrategia de posicionamiento, pues, a través de ella, el autor explicita que su trabajo proviene de un trabajo mayor, que ha sido validado por su comunidad, por lo que, indirectamente, creemos, está intentando validar también el AIC que presenta. Un ejemplo es el siguiente: "Este artículo es un extracto de la Tesis Doctoral que, con el mismo título de este artículo, fue presentada en la Facultad de Teología de la Pontificia Universidad Católica de Chile el 15 de marzo de 2012, bajo la dirección del profesor Sergio Silva Gatica, SS.CC." [Hum_F_1807]. Desde la perspectiva de la autora, con el uso mayoritario de esta estrategia textual de posicionamiento, el autor construye un ethos o perfil de investigador autorizado y protegido, pues destaca que su trabajo es parte de un trabajo mayor, que ya ha sido validado por su comunidad. Este es el caso de los autores expertos de Humanidades, quienes, de hecho, se valen exclusivamente de esta estrategia de la dimensión textual. 
Ahora bien, la estrategia más frecuente en todos los grupos que recurren a la dimensión textual para posicionarse, excepto en los autores consolidados de Humanidades, es Agradecer las revisiones del texto, que, en todos los casos, supera el $50 \%$ de uso al interior de la dimensión en cuestión. Con esta estrategia, el autor, por medio de sus agradecimientos, señala las revisiones o revisores que ha tenido su artículo, posicionando, así, el texto que presenta. Por ejemplo: "Agradezco a Mariano Bertucci, Sebastián Mazzuca, Richard Snyder y dos revisores anónimos por sus muy útiles sugerencias" [CS_C_788]. Para Hyland (2011) el uso de agradecimientos en textos académicos no tendría una función inocente, sino que permitiría al autor mostrarse como un sujeto creíble académicamente mediante la declaración explícita de las personas importantes que conoce en su disciplina y con quienes se relaciona, lo que, en consecuencia, lo involucra en determinadas redes. De esta manera, la inclusión de este tipo de agradecimientos serviría al autor para evidenciar que su texto ha sido revisado por miembros importantes de su comunidad, lo que actúa como una especie de resguardo ante posibles críticas de los evaluadores de su artículo o de los lectores del mismo. A juicio de la autora, a través del uso preferente de esta estrategia, el investigador construye un ethos de autor cauto y con redes o aliados, pues destaca preferentemente las revisiones que ha tenido su texto, las que han sido hechas por miembros importantes de su comunidad. Tal es el caso de los autores de Ciencias Sociales (novatos y expertos) y novatos de Humanidades. El segundo perfil identificado, a partir de la estrategia predilecta de la dimensión textual, es la de investigador autorizado y protegido, propia de autores que destacan que su trabajo es parte de una investigación mayor, que ya ha sido validado por su comunidad. Este es el caso de los autores expertos de Humanidades.

Un caso interesante de comentar es el de Ciencias Sociales, pues los autores novatos y expertos de esta área utilizan solo una estrategia de la dimensión textual, y esa estrategia es exclusiva del grupo. Así, los investigadores en formación solo utilizan "Destacar la importancia de las referencias" y los consolidados solo recurren a "Valorar positivamente su artículo (texto)". La primera estrategia mencionada alude a aquellos casos en que el autor destaca la importancia de las referencias que ha utilizado en su trabajo, mediante afirmaciones como "[las referencias mencionadas] tienen extraordinario interés" [CS_F_1161]. La segunda estrategia aludida refiere a los casos en que el autor alaba su propio artículo, en cuanto producto textual, como se observa en el siguiente ejemplo: "La visión general de los estudios sobre regímenes y democracia en América Latina que presenta este artículo arroja un saldo muy positivo" [CS_C_788].

\section{CONCLUSIONES}

Sobre la base de los resultados de la investigación, se pueden derivar las siguientes seis conclusiones centrales.

Primero, en el corpus total, la dimensión epistémica es la que predomina ampliamente frente a la social y textual, por lo que prima el interés por posicionar el aporte que se hará al conocimiento con el trabajo, por sobre la valoración que pueda hacer de sí mismo como investigador o de las características textuales de su trabajo.

Segundo, las tres dimensiones identificadas (epistémica, social y textual) están presentes en todas las áreas y grados de experticia estudiados, a excepción de los novatos de Ingeniería y Tecnología, grupo en el que no se encontraron estrategias asociadas a la dimensión textual.

Tercero, en todos los estratos analizados se recurre preferentemente a la dimensión epistémica para posicionar las investigaciones.

Cuarto, los escritores novatos de Ciencias Sociales son quienes, en todas las dimensiones, utilizan mayor variedad de estrategias de posicionamiento del autor. En el polo contrario, los novatos de Ingeniería y Tecnología son quienes utilizan menor variedad de estrategias de las tres dimensiones que constituyen la propuesta presentada.

Quinto, los autores y áreas de la ciencia que necesitan ganar reconocimiento son quienes mayor cantidad de estrategias de posicionamiento utilizan. Así, en cuanto a áreas de la ciencia, es Ciencias Sociales la que recurre a mayor cantidad de estrategias, que es un área que ha tenido que pelear por su lugar en investigación; mientras que Ingeniería las utiliza en muy baja proporción, pues ya cuenta con un prestigio social e investigativo. Del mismo modo, en cuanto a grado de experticia, son los autores novatos de las áreas mencionadas quienes utilizan más estrategias de posicionamiento que los expertos.

Finalmente, a partir de la estrategia predilecta para posicionar la investigación, es posible proyectar el ethos del investigador.

La investigación presentada constituye un avance importante en la descripción discursiva del AIC, pues presentamos las estrategias específicas, identificadas a partir de un análisis de corpus, que utilizan los autores para posicionar sus trabajos. Dichas estrategias, además, se presentan de acuerdo con dos variables: área de la ciencia y grado de experticia del autor. En este sentido, los resultados pueden ayudar a cualquier persona interesada en la producción científica, pues les ofrecemos las estrategias concretas utilizadas en su área para posicionar un AIC. 


\section{AGRADECIMIENTOS}

La investigación fue realizada gracias al financiamiento del Proyecto FONDECYT de Postdoctorado №3150056, titulado "El posicionamiento estratégico del autor en la comunicación del conocimiento científico: formas y funciones discursivas de la información propia y ajena en artículos de investigación".

\section{REFERENCIAS}

Abdi, J. y F. Farrokhi, Investigating the Projection of Authorial Identity through First Person Pronouns in L1 and L2 English Research Articles, doi: 0.15640/ijll.v3n1a21, International Journal of Language and Literature, 3(1), 156-168 (2015)

Blessing, L. y A. Chakrabarti, DRM, a Design Research Methodology, 1르. Ed., Springer, Dordrecht, Holanda (2009)

Basturkmen, H., A genre-based investigation of discussion sections of research articles in dentistry and disciplinary variation, doi: 10.1016/j.jeap.2011.10.004, Journal of English for Academic Purposes, 11(2), 134-144 (2012)

Bongiovani, P., N. Gómez y S. Miguel, Opiniones y hábitos de publicación en acceso abierto de los investigadores argentinos. Un estudio basado en los datos de la encuesta SOAP, doi: 10.3989/redc.2012.3.903, Revista Española de Documentación Científica, 35(3), 453-467 (2012)

Bunton, D., Generic moves in PhD thesis introductions, $1^{\text {a }}$ Ed., in Academic discourse by J. Flowerdew, pp.57-75, Pearson, Harlow, Inglaterra (2002)

Estaji, M. y R. Vafaeimehr, A Comparative Analysis of Interactional Metadiscourse Markers in the Introduction and Conclusion Sections of Mechanical and Electrical Engineering Research Papers, https://goo.gl/Ey4N3A, ISSN: 2322-1291, Iranian Journal of Language Teaching Research, 3(1), 37-56 (2015)

Fleiss, J., Measuring Nominal Scale Agreement among many Rater, doi: 10.1037/h0031619, Psychological Bulletin, 76(5), 378-382 (1971)

Gibbons, M., C. Limoges, H. Nowotny, S. Schwartzman, P. Scott y M. Trow, La nueva producción del conocimiento. La dinámica de la ciencia y la investigación en las sociedades contemporáneas, Ediciones Pomares, Barcelona, España (1997)

Gutiérrez, B. El lenguaje de las Ciencias, 1aㅡ Ed., Gredos, Madrid, España (2005)

Hernández, R., C. Fernández, y P. Baptista, Metodología de la investigación, 4ª Ed., McGraw-Hill, México DF, México (2006)

Hyland, K., Projecting an academic identity in some reflective genres, https://goo.gl/2jWNHZ, ISSN: 1139-7241, Ibérica, 21, 9-30 (2011)

Hu, G. y F. Cao, Hedging and boosting in abstracts of applied linguistics articles: A comparative study of English-and Chinese-medium journals, doi: 10.1016/j.pragma.2011.04.007, Journal of Pragmatics, 43(11), 2795-2809 (2011)

Jackson, S., Research Methods and Statistics. A Critical Thinking Approach, 1를., Wadsworth, Belmont, Estados Unidos (2009)

Jiang, F., Stance and voice in academic writing, doi: 10.1075/ijcl.22.1.04jia, International Journal of Corpus Linguistics, 22(1), 85-106 (2017)

Kim, L. y J. Lim, Metadiscourse in English and Chinese Research Article Introductions, doi: 10.1177/1461445612471476, Discourse Studies, 15(2), 129-146 (2013)

Landis, J. y G. Koch, The measurement of observer agreement for categorical data, doi: 10.2307/2529310, Biometrics, 33, 159-174 (1977)

Larivière, V., E. Archambault, Y. Gingras y É. Vignola-Gagné, The place of serials in referencing practices: Comparing natural sciences and engineering with social sciences and humanities, doi: 10.1002/asi.20349, Journal of the Association for Information Science and Technology, 57(8), 997-1004 (2006)

Li, L., y G. Ge, Genre analysis: Structural and linguistic evolution of the English-medium medical research article (19852004), doi: 10.1016/j.esp.2008.12.004, English for Specific Purposes, 28(2), 93-104 (2009)

Loi, C., Research article introductions in Chinese and English: A comparative genre-based study, doi: 10.1016/j.jeap.2010.09.004, Journal of English for Academic Purposes, 9(4), 267-279 (2010)

Martínez, J., y L. Martínez, Determinación de la máxima varianza para el cálculo del factor de imprecisión sobre la escala de medida, y extensión a diferentes tipos de muestreo, Psicothema, 20(2), 311-316 (2008)

Matías-Guiu, J. y R. García-Ramos, Fraude y conductas inapropiadas en las publicaciones científicas, doi: 10.1016/S02134853(10)70015-3, Neurología, 25(1), 1-4 (2010)

Meza, P., La comunicación del conocimiento en el género, Tesis de Lingüística: Comparación entre grados académicos; en Leer y escribir en contextos académicos y profesionales, pp. 67-112, Editorial Planeta, Santiago, Chile (2015)

Meza, P., El posicionamiento estratégico del autor en artículos de investigación: una propuesta para su estudio, doi: 10.15446/fyf.v29n2.60191, Forma y Función, 29(2), 111-134 (2016) 
Meza, P., El posicionamiento estratégico del autor en Artículos de Investigación: un modelo empíricamente fundado, doi: 10.15443/RL2711, Logos: Revista de Lingüística, Filosofía y Literatura, 27(1), 152-164 (2017a)

Meza, P. Modelo posicionamiento estratégico del autor en Artículos de Investigación. (En la web: https://goo.gl/X27CvD, acceso: 07 de mayo de 2017 (2017b)

Meza, P., Criterios de inclusión para la conformación del corpus CORAI ACEX. (En la web: https://goo.gl/pEFxgY, acceso: 07 de mayo de 2017, DOI: 10.13140/RG.2.2.10233.26720 (2017c)

Meza, P., Ciencia y publicidad a través de las disciplinas: estrategias de autopromoción en artículos de investigación de autores consolidados y en formación, https://goo.gl/HEgh4S, ISSN:1577-6921, Tonos Digital, 32, 1-25 (2017d)

Müller, G., Metadiscurso y perspectiva: Funciones metadiscursivas de los modificadores de modalidad introducidos por 'como' en el discurso científico, doi: 10.4067/S0718-09342007000200005, Revista Signos. Estudios de Lingüística, 40(64), 357-387 (2007)

Sabaj, O., K. Matsuda y M. Fuentes, Un modelo para la homogeneización de las clases textuales de la biblioteca electrónica Scielo-Chile: la variabilidad del Artículo de Investigación en diversas disciplinas, doi: 10.4067/S071807642010000600015, Información Tecnológica, 21(6), 133-148 (2010)

Sánchez, A., Manual de Redacción Académica e Investigativa: Cómo escribir, evaluar y publicar artículos, 1a Ed., Cooimpresos, Medellín, Colombia (2011)

Tognini-Bonelli, E. Corpus Linguitics at Work, John Benjamins, Amsterdam, Holanda (2001)

Yotimart, D. y N. Aziz, Linguistic Disadvantage and Authorial Identity in Research Articles Written by Native English and Thai Writers in International Publication, doi: 0.7575/aiac.ijalel.v.6n.5p.206, International Journal of Applied Linguistics and English Literature, 6(5), 206-213 (2017) 